\title{
Focused-Laguerre-Gaussian 3D-Trapping and Spanner at Low Numerical Aperture
}

\author{
Pengcheng Peng ${ }^{1, \mathrm{a}}$, Xintong Chen ${ }^{1, \mathrm{~b}}$, Weizhu Chen ${ }^{1, \mathrm{c}}$, Mingyuan Xie ${ }^{1,2, \mathrm{~d}}$, Fuli Zhao ${ }^{1, \mathrm{e}, *}$ \\ ${ }^{1}$ State Key Laboratory of Optoelectronic Materials and Technologies, Sun Yat-sen University, \\ Guangzhou, 510275, China \\ ${ }^{2}$ School of Information Technology, Beijing Institute of Technology, Zhuhai, \\ Zhuhai, 519088, China \\ a pengpch@mail2.sysu.edu.cn, ${ }^{\mathrm{b}}$ chxint@mail2.sysu.edu.cn, ${ }^{\mathrm{c}}$ chwzhu@mail2.sysu.edu.cn, \\ dxiemingy@mail2.sysu.edu.cn, ${ }^{\mathrm{e}}$ stszfl@mail.sysu.edu.cn \\ *Corresponding author
}

Keywords: 3D-Trapping, Long Axial Trapping, Optical Spanner.

\begin{abstract}
Stable 3D micromanipulation by light requires that gradient force overcome axial scattering force introduced by an objective lens. Although high numerical aperture (NA) objective lenses in conventional optical tweezers could match the requirement, the dramatic limited axial working range and a narrow view field draw back the application seriously. With purpose to improve the application of micromanipulation, we succeed in the three dimensional (3D) trapping of polystyrene microspheres with a low-numerical-aperture $(\mathrm{NA}=0.40)$ objective releasing a long working distance $(\mathrm{WD}=5.89 \mathrm{~mm}$ ) by utilizing the Laguerre-Gaussian beams. A series of rotating manipulation through modulating the asymmetry of Laguerre-Gaussian beams are presented. This work offers an extended axial trapping range for $3 \mathrm{D}$ manipulation and a delicate hand-actuated rotating system for optical manipulation.
\end{abstract}

\section{Introduction}

Optical trapping [1] has been widely used in physics, biologyand other fields [2-6] with the development of laboratory techniques, but the trapping performances vary with different wave-front modulation methods [7]. Optical tweezers [1,8] with a single Gaussian beam for 3D trapping requires that the gradient force overcomes the scattering one, which needs a high numerical aperture microscope objective (typically $\mathrm{NA}>1$ ) to satisfy. However, high NA brings a series of disadvantages such as large spherical aberrations, small field of view, high local heating of trapped sample and short working distance, crippling the potential for optical tweezers to perform relevant innovative biophysical measurements [9]. For the purpose of axial trapping with low NA objective lenses, different approaches have been demonstrated to date. Surface tension, as an additional force, was introduced to balance intensive scattering force $[10,11]$. Annular laser beam generated by two axicons was focused to get a long-distance axial trapping [12]. Single focused-Bessel light beam was employed to obtain 3D optical manipulation [13]. Laguerre-Gaussian (LG) beams was focused with a nonimmersion objective $(\mathrm{NA}=0.8)$ to achieve an axial trapping range of less than $300 \mu \mathrm{m}$ 
[14]. These contributions were significant, but more longer axial trapping range 3D trapping systems are needed for experiments, such as trapping objects at free liquid surface, reducing the influence of substrate while studying the nucleation of crystals and some other special processes. Our single optical trap of long axial trapping range and low-injury required power would be more convenient and flexible in some special biophysical experiments: the translocation of a protein, sensitivity study of protein-mediated DNA loops, single molecule fluorescence techniques $[9,15,16]$. In this letter, we demonstrate the stable 3D trapping and manipulation of polystyrene microspheres with diameter of $8 \mu \mathrm{m}$, using a single LG light beam focused through a microscope objective with low numerical aperture $(\mathrm{NA}=0.40)$, long working distance $(\mathrm{WD}=5.89 \mathrm{~mm})$ and low required trapping power $(\mathrm{P}=3.3 \mathrm{~mW})$.

\section{Experiment}

The experimental layout of our trapping system is shown in Figure 1 (a). A semiconductor laser (Excelsior-532-300-CDRH, Spectra-Physics, Japan) working at wavelength of 532nm is expanded by a telescope formed by Lens 1 and Lens 2, which holds a focal distance of $38.1 \mathrm{~mm}$ and $200 \mathrm{~mm}$ respectively. After beam expanding, the collimated beam passes through the spiral phase plate (SPP) to be transformed into the LG beam. Then LG beam is reflected by a dichroic mirror (short-pass $532 \mathrm{~nm}$ ) into the back aperture of a long working distance $20 \times$ objective (L Plan, NA 0.40, $5.889 \mathrm{~mm}$ working distance, Daheng Optics Inc., China). The sample is mounted on a motorized XYZ stage (DRVER Model, New Focus Inc., USA) that can be driven manually or by a programmable software interface. A condenser was used to converge the red light produced by the illumination (GCI-0604 LED, wavelengh $621 \mathrm{~nm}$, Daheng Optics Inc., China) into sample cell. A USB CCD camera (MC20-C, $6.45 \times 6.45 \mu \mathrm{m}$ pixel size, Micro-shot Technology Inc., China) is employed to record the trapping process. The CCD camera allows imaging speed as high as $15 \mathrm{fps}$ at a resolution of $1360 \times 1024$ pixels. A long-pass filter (long-pass $550 \mathrm{~nm}$ ) is inserted in front of the CCD camera to block the trapping laser beam. The maximal laser power delivered on the sample is about $10 \mathrm{~mW}$. Polystyrene microspheres (8um in diameter, DAE Scientific Inc., China) immersed in water in a sample chamber are used for optical trapping and manipulation. In order to verify the reliability of our SPP, the interference pattern of LG beam (topological charge $l=2$ ) and the plane wave as shown in Figure 1 (b), Figure 1 (c) shows the interference pattern of LG beam (topological charge $l=2$ ) and the spherical beam.

To analyse trapping stiffness of our optical tweezers system, we calculate the escape force of 3D trapped beads based on Stokes' law. Then, we considered a series of generalized LG laser beams by moving SPP perpendicular to the direction of beam propagation (laterally). In this situation, the output modulated LG beams lose axial symmetry, and we performed a set of rotation manipulation based on this precisely controlled asymmetry, proving that the rotation rate of polystyrene microspheres increases with increasing the asymmetry parameter in a certain range. Because of sufficient working distance and a large lateral stiffness, the particles can be fully controlled in three dimensions and, moreover, it can be moved across the entire sample cell at the trapped situation. Our work offers an advanced mechanical rotating system for optical manipulation and a long axial trapping range for 3D optical manipulation, a large field of view, low local heating of trapped samples, and reduced spherical aberrations compared with typical high NA 3D trapping systems. All measurements were carried out at room temperature. 


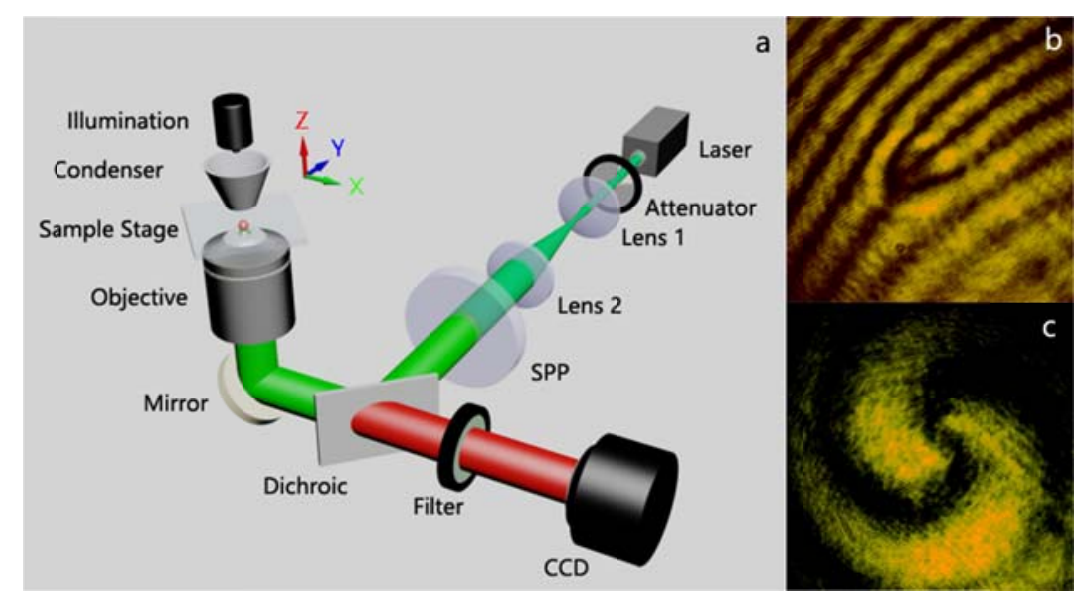

Figure 1 (a) Experimental layout of the 3D trapping and manipulation system. (b) Interference pattern of plane wave and LG beam with topological charge of $l=2$. (c) Interference pattern of spherical beam and the same LG beam.

\section{Results and discussion}

\subsection{Trapping}

As we know, LG beam has special optical vortices, which can be interpreted as its eigen mode of the paraxial Helmholtz equation:

$$
u_{p}^{l}(r, z)=(-1)^{p}\left(\frac{\sqrt{2} r}{w}\right)^{l} L_{p}^{l}\left(\frac{2 r^{2}}{w^{2}}\right) \exp \left(-\frac{r^{2}}{w^{2}}\right)
$$

where $L_{p}^{l}(x)$ is a generalized Laguerre polynomial with radial index $p, l$ is the topological charge, and $w$ is the beam radius [17]. The $p=0$ modes used in our experiment form a series of single annular rings with different topological charges, which related to the number of $2 \pi$ cycles in azimuthal phase around the ring and give rise to the radius of peak intensity of our single annular ring. In our trapping system, laser beam are expanded to match the SPP and fit the back aperture of objective lens. The minimal required force $F_{\min }$ to trap a particle is given by [18]

$$
F_{\text {min }}=\frac{\pi}{6}\left(\rho_{s}-\rho_{m}\right) d^{3} g+\frac{2 k T}{d}
$$

where $\rho_{s}$ is the density of a particle, $\rho_{m}$ is the density of the surrounding fluid, $d$ is this particle diameter, $g$ is the gravitational acceleration, and $T$ is the ambient temperature. The second term of this equation relates to the force required to conquer the Brownian motion. To obtain 3D optical trapping, the gradient force produced by focused LG beam has to be large enough to overcome both Brownian motion and the scattering force in axial direction, and only Brownian motion in lateral direction, which guided our whole experiment process. We achieved stiff 3D trapping and long axial manipulation capabilities of focused LG beam for polystyrene microspheres with different topological charge at low numerical aperture. In order to eliminate the influence of sample cell bottom, we started trapping polystyrene microspheres with a balanced power at an equilibrium position which is about $10 \mu \mathrm{m}$ distance above the bottom layer. To find the balanced power, we adjusted the beam attenuator and realized the most clearly imaging of the trapped particle in CCD system. And then, we moved sample stage sideways and along the vertical axis, showing that the trapped particle kept its spatial position constant. Here, we show that trapped particle can be manipulated in three dimensions. An assumed geometric point adhered to the bottom of sample cell 
was used as reference origin of three dimensional Cartesian coordinates and unit coordinate value equals $1 \mu \mathrm{m}$. Trapped particles surrounded by white doughnut in Figure 2 kept sharpness and position unaltered. The first row labelled by trapped particle $\mathrm{x}$ coordinate value of 0,10 , and 20 , respectively, shows trapped sphere kept its sharpness and spatial position invariable in the $\mathrm{x}$ direction when sample stage was moved along $\mathrm{x}$ axis. Similarly, the second row shows trapped particle kept its sharpness and spatial position fixed on y axis. Here, lateral trapping of focused LG beam was demonstrated fully. The third row demonstrates stable axial trapping process and is labelled by trapped particle $\mathrm{z}$ coordinate value of 0,30 , and 60 , which is the key for $3 \mathrm{D}$ optical trapping. In the axial direction, namely $\mathrm{z}$ axis, a trapped bead was in equillibrium condition of gravity, flotage, scattering force and gradient force. The flotage and scattering force kept the same direction absolutely. Gradient force pointed to the focus of objective lens, and its size or direction depended on equilibrium position of trapped particle, associating with the power and topological charge of incident LG beam.

Figure 3 shows 3D trapping power distribution of LG beam with topological charge varies from $2-16$, and laser power was all measured in the plane of sample cell. To be 3D trapped, bead must be in an equilibrium range of optical trap in axial direction especially. Therefore, balanced power has a closed scope of value, which is marked by error bar in Figure 3 for different topological charges. Real points indicate the most appropriate balanced power, and trapped particle kept the highest resolution in CCD system. With the increase of topological charge, balanced power increases approximatively linearly. The reason is that with increasing topological charge value, the size of bright annulus in LG modes increases, with fewer scattering light ray holding the bead and a smaller fraction of light field interacts with the trapped sphere of diameter $=8 \mu \mathrm{m}$ [19]. In addition, closed range of balanced power value decrease, this is due to the fact that axial thickness of bright annulus in LG modes, or axial trapping well, decreases with increasing topological charge.

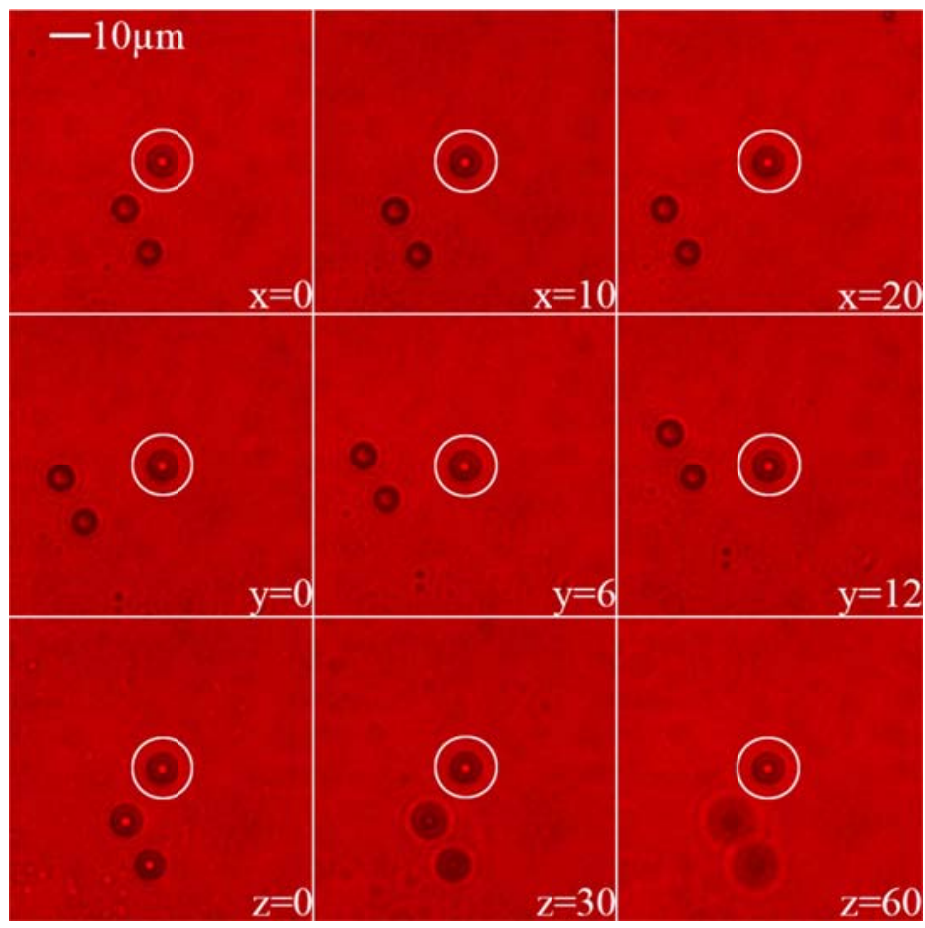

Figure 2 Polystyrene sphere of $8 \mu \mathrm{m}$ diameter trapped by the focused LG mode with topological charge $l=2$ and fixed power $\mathrm{P}=3.3 \mathrm{~mW}$ while sample stage is moved along coordinate $\mathrm{x}, \mathrm{y}, \mathrm{z}$.

Spheres resting at the bottom of sample cell provide a visual reference. 


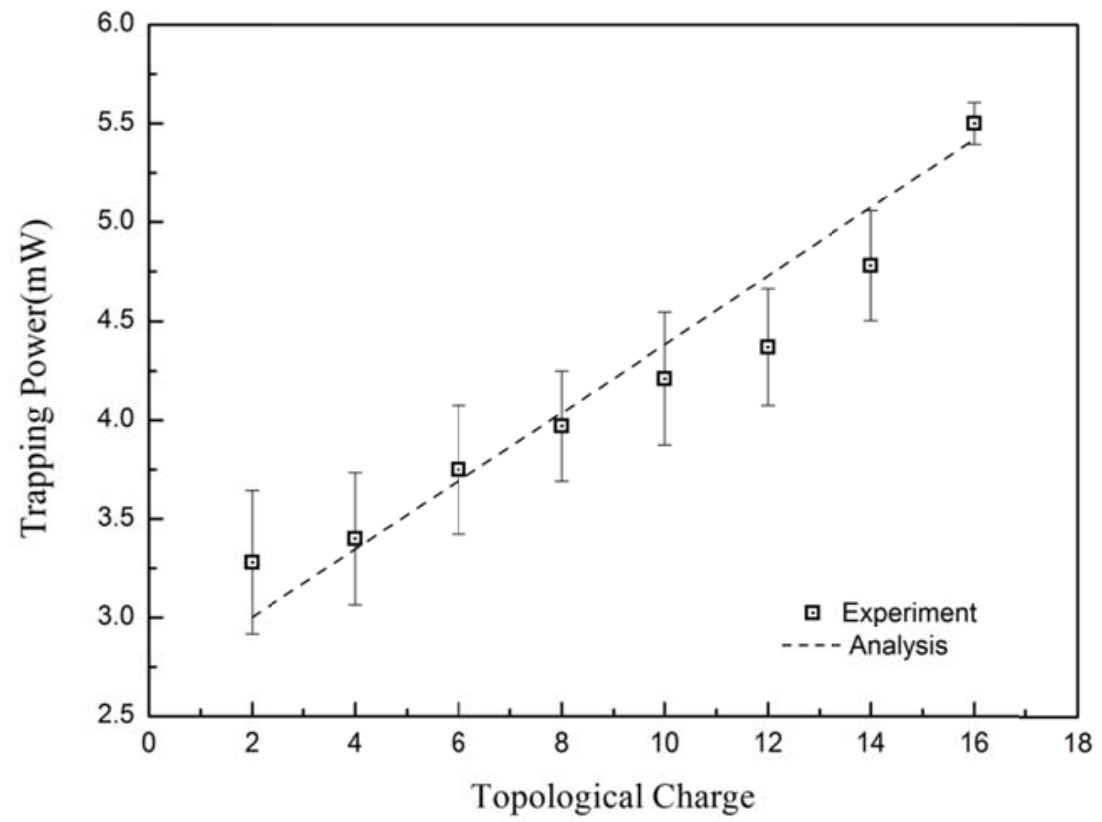

Figure 3 Comparison between the experimental measurements and linear analysis for stable 3D trapping.

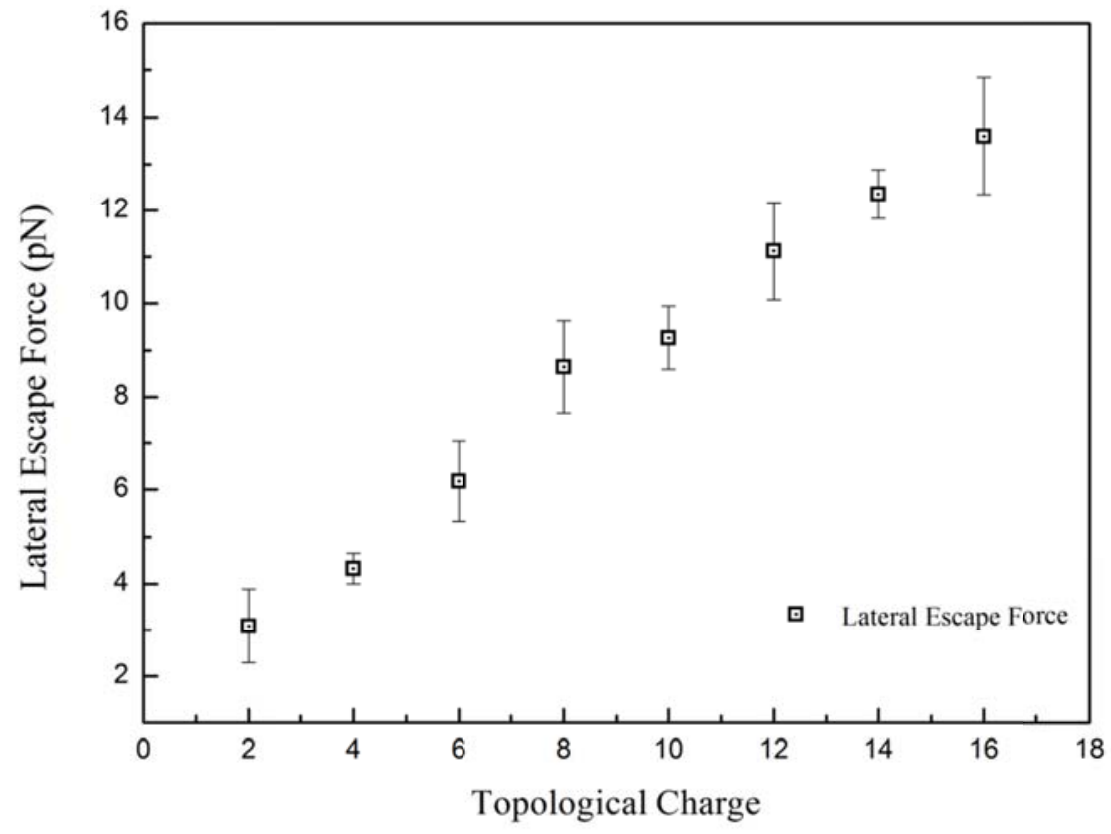

Figure 4 Lateral escape force for individual 3D trapped bead ( $8 \mu \mathrm{m}$ diameter) as a function of different topological charge.

To evaluate lateral trapped stiffness of different LG modes, we drive the motorized stage in x-y plane in a certain velocity when 3D trapped individual bead escaped along lateral direction exactly corresponding to required power in Figure 3. We got the escape velocities for different topological charges and calculated the escape force from Stokes' law [20]

$$
F=6 \pi \eta r v
$$


where $\eta$ is the viscosity of solution, $r$ is the radius of bead, and $v$ is the drag velocity. The lateral escape force was found to increase with topological charge showed in Figure 4. The average light force generated by our optical tweezers system in lateral direction was calculated to be $2.06 \mathrm{pN} / \mathrm{mW}$, which shows a well trapping performance though it works at an extremely low numerical aperture.

\subsection{Spanner}

Like standard LG mode, the asymmetric LG beam (aLG-beam) has transverse intensity distribution consists of a finite number of light ring, but the intensity distribution along the ring is not uniform, which has a crescent shape and rotates while propagating and can be used to rotate large asymmetrical microparticles [21,22]. In particular, the normalized orbital angular momentum (OAM) of aLG-beam depends on the beam topological charge linearly and on asymmetry index parabolically [22].

To realize a simple mechanical transmission optical spanner, we generated aLG-beam through steps of shifts in Cartesian coordinates compared with conventional LG mode. The aLG-beam was generated by misplaced SPP with a shift between the SPP centre and incident Gaussian beam. Then we achieved different rotational velocity manipulation in focus of the aLG-beam through changing the shift. We show that the velocity of microspheres increases within limit with increasing this special asymmetry index and invariable topological charge, which coincides with Ref. [22]. Figure 5 shows rotation frames of two particles in focus of the aLG-beam with $0.75 \mathrm{~mm}$ shift, $l=2$ topological charge of SPP and $3.3 \mathrm{~mW}$ laser power. The average rotation velocity of particles equals $0.22 \pm 0.03 \mu \mathrm{m} / \mathrm{s}$. Figure 6 shows experimental rotation angular (dash line) and corresponding average angular velocities (solid line). When the shift is $0.75 \mathrm{~mm}$, rotation amplitude and rotation velocity achieve the maximal value. Therefore, we could precisely control the rotation manipulation of trapped particles by manually controlling the spatial positon of SPP. It is a simple and effective means to implement optical rotation manipulation.

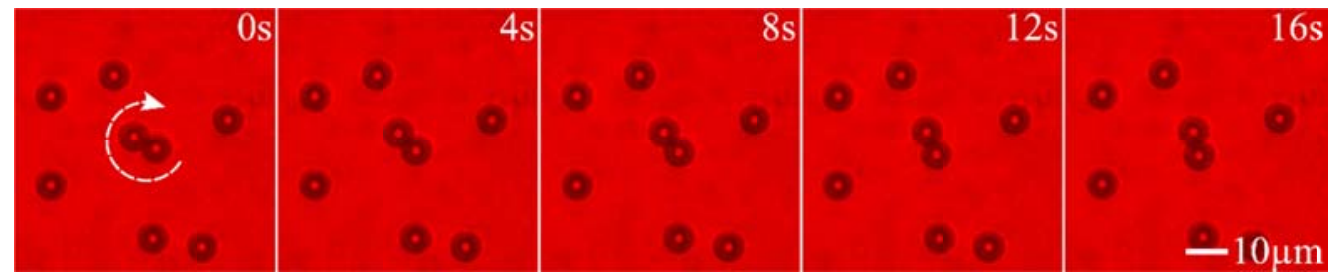

Figure 5 Rotation manipulation of two $8 \mu \mathrm{m}$ diameter particles with asymmetric LG mode whose topological charge $l=2$. 


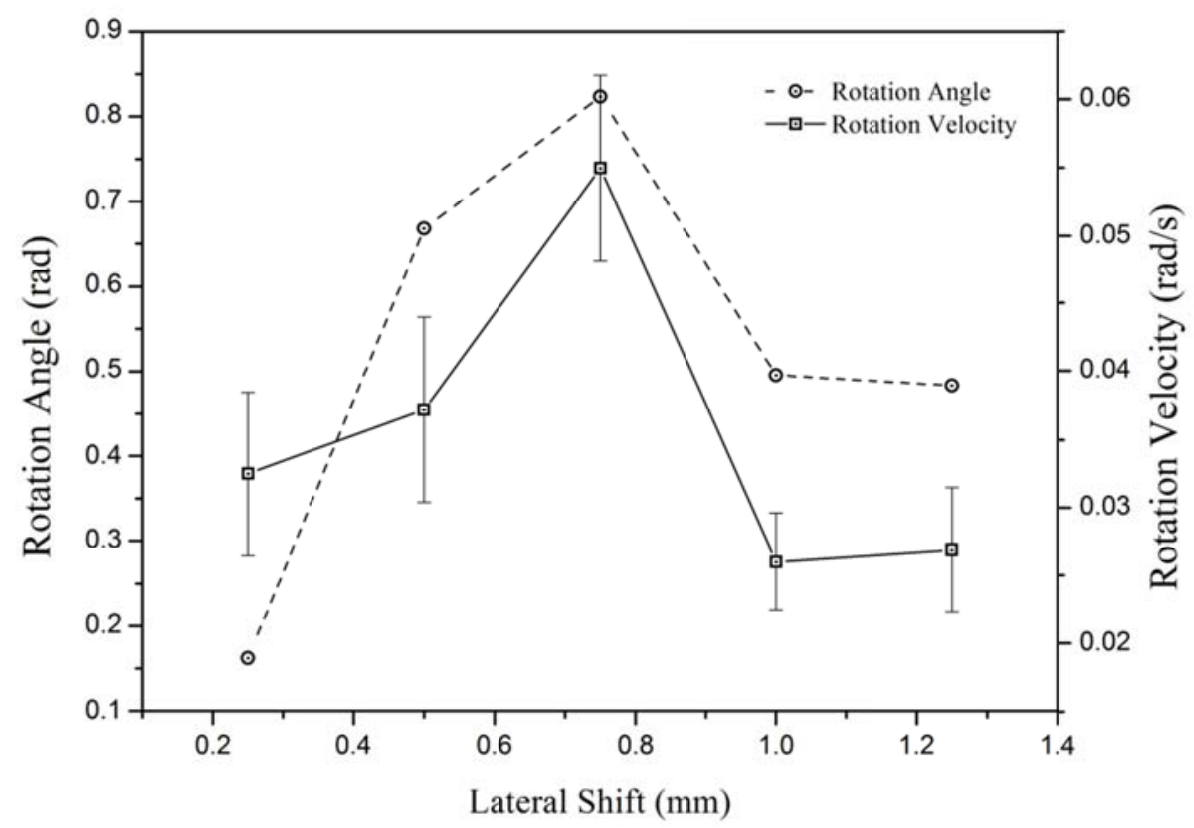

Figure 6 The rotation angle and average rotation velocity of two trapped samples at different asymmetry index.

\section{Conclusion}

We have presented a feasible stable 3D trapping and manipulation system with a single Laguerre-Gaussian beam focused by a low numerical aperture of NA $=0.40$ compared with conventional optical tweezers for 3D trapping. Based on the long axial trapping distance of $\mathrm{WD}=5.89 \mathrm{~mm}$ and high modulation index of SPP, the trapped particles can be moved throughout the whole sample cell and be hold for several hours stiffly, which facilitates the calibration and operation of 3D optical trapping. Our experimental setup was free from the complicated trapping beams, SLM, or other external forces, with only the SPP. Such an optical tweezers might find its significant applications, since it offers a larger field of view, and lower spherical aberrations.

\section{Acknowledgements}

This work was supported by the National Natural Science Foundation of China (11274397), the Government of Guangdong Province for NSF (2016B090918099) and grants from the Shanghai Institute of Optics and Fine Mechanics (SIOM) and the Chinese Academy of Sciences (CAS).

\section{References}

[1] A. Ashkin, J. M. Dziedzic, J. E. Bjorkholm, and Steven Chu. (1986) Observation of a Single-beam Gradient Force Optical Trap for Dielectric Particles. Optics Letters, 11,5.

[2] Jeffrey R. Moffitt, Yann R. Chemla, Steven B. Smith, and Carlos Bustamante. (2008) Recent Advances in Optical Tweezers. Annu. Rev. Biochemistry, 77, 205-28.

[3] T Cizmar, L C Davila Romero, K Dholakia, and D L Andrews. (2010) Multiple Optical Trapping and Binding: New Routes to Self-assembly. J. Phys. B: At. Mol. Opt. Phys., 43, 102001.

[4] Mike Woerdemann, Christina Alpmann, Michael Esseling, and Cornelia Denz. (2013) Advanced Optical Trapping by Complex Beam Shaping. Laser Photonics Rev., 7, 6, 839-854.

[5] Timo A. Nieminen, Nathaniel du Preez-Wilkinson, Alexander B. Stilgoe, Vincent L.Y. Loke, Ann A.M. Bui, HalinaRubinsztein-Dunlop. (2014) Optical Tweezers: Theory and Modelling. Journal of Quantitative Spectroscopy \&Radiative Transfer, 146, 59-80. 
[6] Yann R. Chemla. (2016) Review High-Resolution, Hybrid Optical Trapping Methods, and Their Application to Nucleic Acid Processing Proteins. Biopolymers, 105, 10.

[7] David G. Grier. (2003) A Revolution in Optical Manipulation.Nature, 424, 14.

[8] A. Ashkin, J. M. Dziedzic \& T. Yamane. (1987) Optical Trapping and Manipulation of Single Cells Using Infrared Laser Beams. Nature, 330, 24-31.

[9] Samuel Yehoshua, Russell Pollari, and Joshua N. Milstein. (2015) Axial Optical Traps: a New Direction for Optical Tweezers. Biophysical Journal, 108, 2759-2766.

[10] Alexander Jesacher, Severin Furhapter, Christian Maurer, Stefan Bernet, and Monika Ritsch-Marte. (2006) Holograhic Optical Tweezers for Object Manipulations at an Air-Liquid Surface. Optics Express, 14, 13.

[11] R Dasgupta, S Ahlawat and P K Gupta. (2007) Trapping of Micron-Sized Objects at A Liquid-Air Interface. Journal of Optics A: Pure and Applied Optics, 9, 189-195.

[12] Ming Lei, Ze Li, Shaohui Yan, Baoli Yao, Dan Dan, Yujiao Qi, JiaQian, Yanlong Yang, PengGao, Tong Ye. (2013) Long-Distance Axial Trapping with Focused Annular Laser Beams.Plos One, 8, 3.

[13] Yareni A. Ayala, Alejandro V. Arzola, and Karen Volke-Sepulveda. (2016) 3D Micromanipulation at Low Numerical Aperture with a Single Light Beam: the Focused-Bessel Trap. Optics Letters, 41, 3.

[14] RaktimDasgupta, Ravi Shanker Verma, SunitaAhlawat, Deepa Chaturvedi, and Pradeep Kumar Gupta.(2011) Long-Distance Axial Trapping with Laguerre-Gaussian Beams. Applied Optics, 50, 10.

[15] Yih-Fan Chen, J. N. Milstein, and Jens-Christian Meiners. (2010) Protein-Mediated DNA Loop Formation and Breakdown in a Fluctuating Environment.Physical Review Letters, 104, 258103.

[16] BhanuNeupane, Fang Chen, Wei Sun, Daniel T. Chiu, and Gufeng Wang. (2013) Tuning Donut Profile for Spatial Resolution in Stimulated Emission Depletion Microscopy. Review of Scientific Instruments, 84, 043701.

[17] L. Allen, M. W. Beijersbergen, R. J. C. Spreeuw, and J. P. Woerdman. (1992) Orbital Angular Momentum of Light and the Transformation of Laguerre-Gaussian Laser Modes. Physical Review A, 45, 11.

[18] N. B. Simpson, D. Mcgloin, K. Dholakia, L. Allen and M. J. Padgett. (1998) Optical Tweezers with Increased Axial Trapping Efficiency. Journal of Modern Optics, 45, 9.

[19] Jennifer E. Curtis and David G. Grier. (2003) Structure of Optical Vortices. Phys. Rev. Letter. 90, 133901.

[20] Robert M. Simmons, Jeffrey T. Finer, Steven Chu, and James A. Spudich. (1996) Quantitative Measurements of Force and Displacement Using an Optical Trap. Biophysical Journal, 70, 1813-1822.

[21] Cathering M. Herne, Kristina M. Capzzi, Emily Sobel, and Ryan T.Kropas. (2015) Rotation of Large Asymmetrical Absorbing Objects by Laguerre-Gaussian Beams. OpticsLetters, 40, 17.

[22] Alexey A. Kovalev, Victor V. Korlyar, and Alexey P. Porfirev. (2016) Optical Trapping and Moving of Microparticles by Using Asymmetrical Laguerre-Gaussian Beams. Optics Letters, 41, 11. 